\title{
Long-term outcome of high-dose-rate brachytherapy and perioperative brachytherapy in early mobile tongue cancer
}

\author{
Mahadev Potharaju, MD', Hemanth Raj E, PhD², Manavalan Muthukumaran, PhD', Murali Venkataraman, PhD', \\ Bhargavi llangovan, DNB', Selvan Kuppusamy, BScl \\ 'Department of Radiation Oncology, Apollo Specialty Hospital, Chennai, ${ }^{2}$ Department of Surgical Oncology, Apollo Specialty Hospital, \\ Chennai, India
}

\begin{abstract}
Purpose: To evaluate long-term outcome of high-dose-rate brachytherapy and perioperative brachytherapy in early mobile tongue cancer.

Material and methods: Seventy-three patients with clinically staged T1/T2 N0 M0 of mobile tongue cancer were studied retrospectively. Between January 2000 and September 2010, 47 patients underwent high-dose-rate brachytherapy (HDR-BT) alone and 26 patients underwent perioperative brachytherapy (PB). Endpoints were overall survival, disease-free survival, loco-regional control, and late side effects.

Results: Median age was 52 years and median follow-up was 74 months (range, 60-180). There were no local recurrences in the PB group. Overall survival at 6 years was $74.7 \%$ vs. $92.3 \%$ in HBR BT and PB group, respectively $(p=0.032)$. Disease-free survival at 6 years was $55.3 \%$ vs. $92.3 \%$ respectively in HDR-BT and PB $(p=0.002)$. Disease-free survival at 6 years in tumor histologic grade $1 / 2$ patients was 76.3 months versus $40 \%$ in grade 3 patients. Nodal recurrence-free rate at 6 years was $67.5 \%$ with HDR-BT only, and $96.2 \%$ with PB ( $p=0.007)$. In HDR BT only group, nodal recurrence-free rate at 6 years in T1 patients was $89.8 \%$ versus $29.4 \%$ in T2 patients. $16 \%$ and $7 \%$ patients developed soft tissue necrosis and osteoradionecrosis, respectively. Multivariate Cox proportional hazards analysis revealed significant correlation of local recurrence with tumor grade $(p=0.029)$, nodal recurrence with T-stage $(p=0.007)$, and disease-free survival with age $(p=0.003)$ and T stage $(p=0.026)$.

Conclusions: HDR-BT alone gives acceptable loco-regional control in T1 tumors. T2 stage tumors should not be treated by brachytherapy alone in view of high failure rates in nodal regions and should undergo either neck dissection or nodal irradiation. Perioperative brachytherapy is investigational and can be considered in patients who are at highrisk for local recurrence in patients undergoing surgery alone.

J Contemp Brachytherapy 2018; 10, 1: 64-72 DOI: https://doi.org/10.5114/jcb.2018.74139
\end{abstract}

Key words: head and neck, HDR, perioperative brachytherapy, tongue cancer.

\section{Purpose}

The worldwide annual oral cancer burden is estimated to be around 275,000 of cases, with two-third of the cases coming from developing countries, and tongue is the commonest subsite [1,2]. Early tongue cancer management initiates heated debate among surgical and radiation oncologists. It can be managed by single modality treatment with either surgery or radiotherapy. Surgery is preferred by many because of the short treatment duration, and absence of long-term side effects associated with radiotherapy. The surgical treatment options range from wide local excision of tongue primary with or without neck dissection. Radiotherapy techniques mainly involve brachytherapy and/or external beam radiotherapy. Primary tongue treatment is less controversial, as both surgery and brachytherapy provide good local control [3,4]. Clinically negative neck is a therapeutic dilemma with various options available like observation, elective supraomohyoid/extended neck dissection, or prophylactic radiotherapy to neck. A landmark prospective randomized trial by $\mathrm{D}^{\prime} \mathrm{Cruz}$ et al. showed better overall survival at 3 years in patients undergoing elective neck dissection, compared to therapeutic neck dissection $(80 \%$ vs. $67.5 \%)$ in early tongue cancer management [5]. The 5-year survival rates with primary surgery alone in early oral tongue cancer varies from 70 to $89 \%$. The main reason for failure is loco-regional recurrence rather than distant metastasis. The local and nodal recurrence
Address for correspondence: Mahadev Potharaju, MD, Department of Radiation Oncology, Apollo Specialty Hospital, 320 Anna Salai, Chennai-600035, India, phone: +91 9003239303, ๑e-mail: mahadev62@gmail.com
Received: 30.08.2017 Accepted: 11.02.2018 Published: 28.02.2018 
rates after surgery alone vary from $11-63 \%$ and $18-25 \%$, respectively $[6,7,8,9,10]$. The local recurrence rates after surgery alone for early tongue cancer is not insignificant. In countries where regular long-term follow-up is an issue, not detecting early local recurrence may have devastating consequence. Our aim of adding brachytherapy to the tumor bed at the time of surgery was to estimate whether we can prevent local recurrence. Despite the good results achieved with either surgery or radiotherapy, there is a scope for improvement. The objective of our study was to analyze retrospectively our results with high-dose-rate brachytherapy (HDR-BT) only to the tongue and perioperative brachytherapy (PB). PB involved wide local excision of tongue primary with modified radical neck dissection and placement of brachytherapy tubes at the time of surgery for HDR brachytherapy to tumor bed a week later.

\section{Material and methods}

As per the 2009 criteria of the Union for International Cancer Control (UICC) TNM classification, $17^{\text {th }}$ edition [11], there were 44 patients with T1N0M0 and 29 patients with T2N0M0 (Table 1). Between January 2000 and September 2010, 47 and 26 patients with clinically staged T1/ T2 N0 M0 of the mobile tongue cancer were treated with HDR-BT and PB, respectively. Minimum follow-up was 6 years. No prophylactic neck treatment was completed in HDR-BT patients. All PB patients had an ipsilateral modified radical neck dissection.

All patients were treated as inpatients through the entire course of brachytherapy. A complete dental evaluation was done prior to admission. Loose teeth were extracted at least 10 days prior to implant and grinding was done for sharp teeth. A diagnostic computed tomography $(\mathrm{CT})$ scan of neck was completed in 52 patients prior to the procedure. A neck ultrasound was done in the remaining patients. In HDR-BT patients, clinical target volume (CTV) was assessed by palpation under general anesthesia during the procedure. The CTV was the palpable tumor with $5 \mathrm{~mm}$ margin. HDR-BT was not offered when CTV exceeded $1 \mathrm{~cm}$ in thickness. Therefore, only a single plane implant was placed. A single plane implant is intended to treat a tissue slab of $1 \mathrm{~cm}$ thickness ( $0.5 \mathrm{~cm}$ on either side of the plane implant), according to the Manchester/ Paris implant rules. The same concept was adapted here too, but implant rules of either system was not strictly followed, with respect to the distance between the tubes implanted or the closing of ends of the implant. To compensate non-closing, the tubes at the dorsum side was fixed with two buttons, so that the first dwell position was beyond the intended treatment volume to ensure full dose on the dorsum of the tongue. Inferiorly, care was taken to ensure there was no dose to skin at entry point of catheters. The planning was completed using target point optimization method, especially for the implants planned using CT images (where the target could be drawn), while for the implants planned with orthogonal images, distance optimization (optimization on distance dose points) was used to treat a slab of $5 \mathrm{~mm}$ on either side of the plane of implant. This was done by describing the dose points (for optimization) at $5 \mathrm{~mm}$ from the respective catheters and dwell positions used. T2 tumors accepted for brachytherapy were $3 \mathrm{~cm}$ or less in antero-posterior direction. The spacing between the tubes was $1 \mathrm{~cm}$. Most needles of anterior/posterior were placed $1 \mathrm{~cm}$ anterior and posterior to the palpable tumor. In patients undergoing $\mathrm{PB}$, the tubes were placed in the tongue tumor bed during the surgery with a $1 \mathrm{~cm}$ margin antero-posteriorly for perioperative brachytherapy. All brachytherapy insertions were single-plane implants, as the CTV was always $\leq 1 \mathrm{~cm}$ in thickness. A non-looping straight tube technique was used to place the brachytherapy tubes. Metallic needles were inserted through the submandibular approach, pushing through the floor of mouth and through the center of CTV, and brought on to the dorsum of tongue. The plastic tubes were threaded through the metallic tube intraorally and pulled out retrograde exiting through the submandibular region medial to the mandible.

Orthogonal X-ray-based planning was completed in 56 patients and CT based planning was done in 17 patients (Figure 1). Thin dummy metal wires were inserted into the catheters before scanning or taking the orthogonal films for catheter reconstruction. For accurate reconstruction of each individual catheter, a $2 \mathrm{~mm}$ slice thickness was used in CT based planning. The catheters were identified by flagging each catheter with a label number to ensure same numbering of catheters used in the treatment planning system was reproduced during treatment delivery. Before delivery of each fraction, a dummy run was done to ensure there were no kinks in the catheter path. After completing each fraction, the implant tubes were closed with mandrins to prevent kinking of catheters and keep the passage clean. The prescribed dose was 50 Gy and 40 Gy in HDR-BT and PB groups, respectively. The treatment was delivered on 5 consecutive days, twice a day, with a minimum of 6 hours inter-fraction interval. The treatment in HDR-BT patients started on the same evening of implantation. The PB group was planned for brachytherapy a week after the surgery. All patients completed the planned course of treatment without any interruption.

For CT-based planning, cumulative and differential dose volume histograms were used to evaluate implant

Table 1. Patient and disease characteristics

\begin{tabular}{lcc} 
Factor & HDR-BT $^{\mathrm{a}}$ & $\mathrm{PB}^{\mathrm{b}}$ \\
\hline Number & 47 & 26 \\
\hline Stage & 30 & 14 \\
\hline $\mathrm{T} 1$ & 17 & 12 \\
\hline $\mathrm{T} 2$ & 31 & 18 \\
\hline Male & 16 & 8 \\
\hline Female & 16 & 8 \\
\hline Smokers & 7 & 5 \\
\hline Tobacco chewers & 10 & 6 \\
\hline $\begin{array}{l}\text { Sharp tooth } \\
\begin{array}{l}\text { aHigh-dose-rate brachytherapy } \\
\text { bPerioperative brachytherapy }\end{array}\end{array}$ &
\end{tabular}




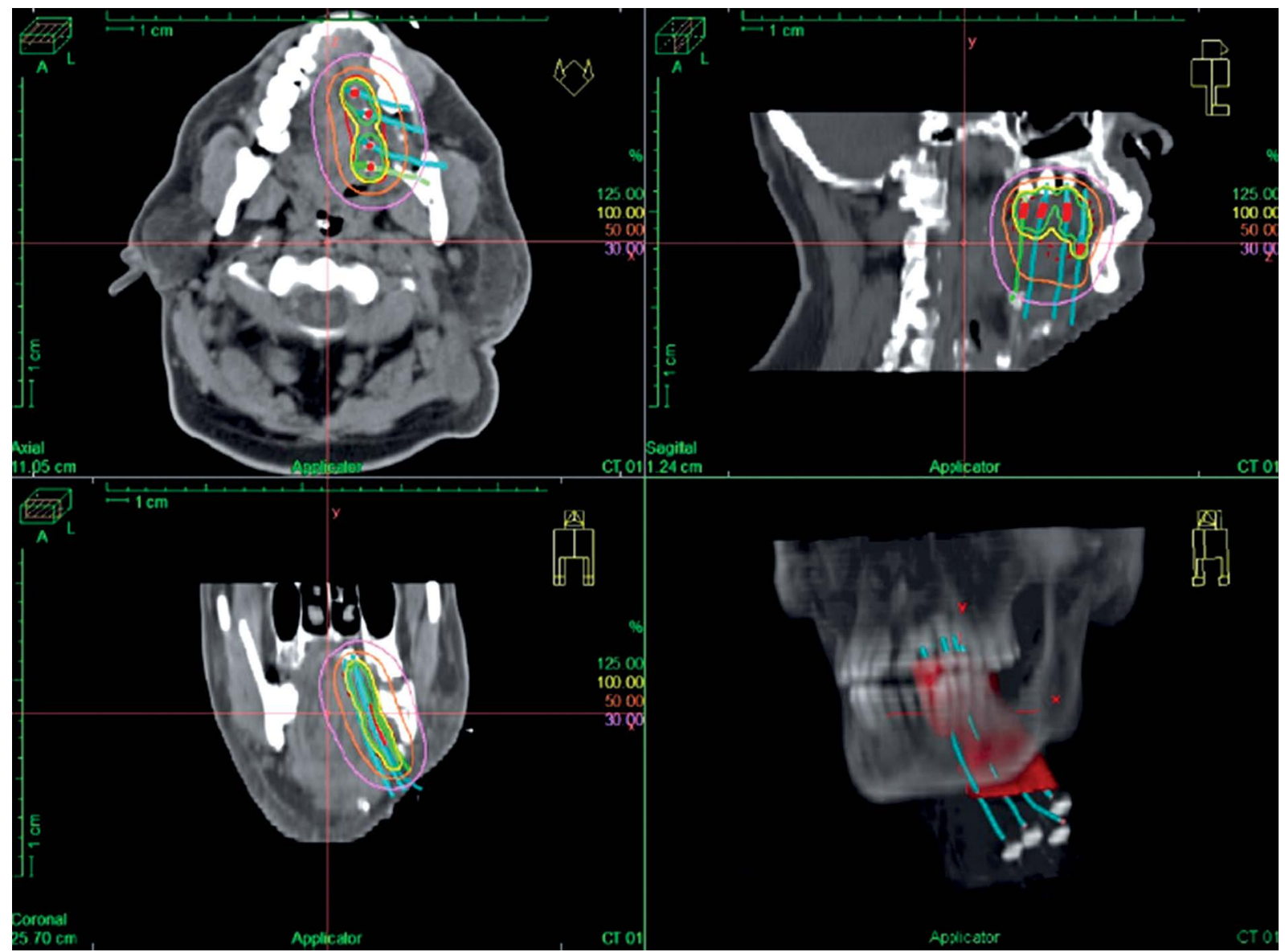

Fig. 1. Axial, sagittal, 3D-reconstruction, and coronal images of high-dose-rate brachytherapy implant (images clockwise from top left)

quality with the help of coverage index (CI), uniformity index (UI), and quality index (QI). CI was defined as a fraction of target volume receiving a dose equal to or greater than reference dose, and ideally reaching 1 . UI was defined as the volume between the dose values of prescribed dose (PD) and high dose (HD), normalized to the $\mathrm{u}$-interval $\left(\mathrm{u}=\mathrm{D}^{-3 / 2}\right)$ between $\mathrm{PD}$ and $\mathrm{HD}$, divided by the volume encompassed by PD, normalized to the $u$ interval between PD and infinity dose. QI was defined as the volume between the dose values of low dose (LD) and high dose (HD), normalized to the $\mathrm{u}$-interval $\left(\mathrm{u}=\mathrm{D}^{-3 / 2}\right)$ between LD and HD, divided by the volume encompassed by LD, normalized to the $u$ interval between LD and infinity dose. QI measures dose homogeneity and UI evaluates how well the target is covered by the prescribed dose. The volume of tissue receiving $150 \%\left(\mathrm{~V}_{150}\right)$ of the prescribed dose was calculated for all patients.

Median target volume surrounded by the chosen isodose was $18.5 \mathrm{~cm}^{3}$. A single planar implant was done with the after-loading catheters placed through submandibular approach. The median number of catheters placed was 5 (range, 4-7). No mandibular shield was used. Endpoints were local-regional control, disease-free survival, overall survival, and late side-effects.

The follow-up protocol was clinical examination every 6 weeks for the first 6 months, every 3 months for 3 years, every 6 months up to 5 years, and annually there- after. The diagnosis of mandibular or soft tissue necrosis was made if there was non-healing exposed bone or soft tissue necrosis for 3-6 months without evidence of clinically recurrent tumor.

The statistical software SPSS version 20.0 for windows (SPSS Inc., Chicago, IL, USA) was used for statistical analysis. The Kaplan-Meir method was applied to calculate the survival and the loco regional control rates. The endpoint was the duration from the start of treatment to the occurrence of the event or last follow-up. The log-rank test was used to assess the statistical significance of the differences between survival curves. Univariate and multivariate Cox proportional Hazards regression analysis was performed for significant predictors of loco regional recurrence and disease-free survival. A $p$ value $<0.05$ was considered statistically significant.

\section{Results}

Forty-seven patients underwent Ir-192 HDR-BT as the primary modality of treatment, with surgical salvage for local and nodal recurrences (Table 2). Twentysix patients underwent $\mathrm{PB}$.

The median age was 52 years, and the median follow-up was 74 months (range, 60-180). In HDR-BT group, local recurrence-free rate at 6 years in tumor histologic grade 1 and 2 patients was $89.7 \%$ vs. $62.5 \%$ in grade 3 pa- 
Table 2. Distribution of treatment failures

\begin{tabular}{lccccc} 
Failure & Stage $1(n=44)$ & Stage $2(n=29)$ & HDR-BT $^{\mathrm{a}}(n=47)$ & $\mathrm{PB}^{\mathrm{b}}(n=26)$ & Total $(n=73)$ \\
\hline Local & 3 & 4 & 7 & 0 & $7(9.6 \%)$ \\
\hline Nodal & 6 & 10 & 15 & 1 & $16(22 \%)$ \\
\hline Local \& nodal & 0 & 2 & 2 & 0 & $2(2.7 \%)$ \\
\hline Distant & 0 & 1 & 0 & 1 & $1(1.3 \%)$ \\
\hline Total & 9 & 17 & 24 & 2 & $26(35.6 \%)$
\end{tabular}

aHigh-dose-rate brachytherapy bPerioperative brachytherapy
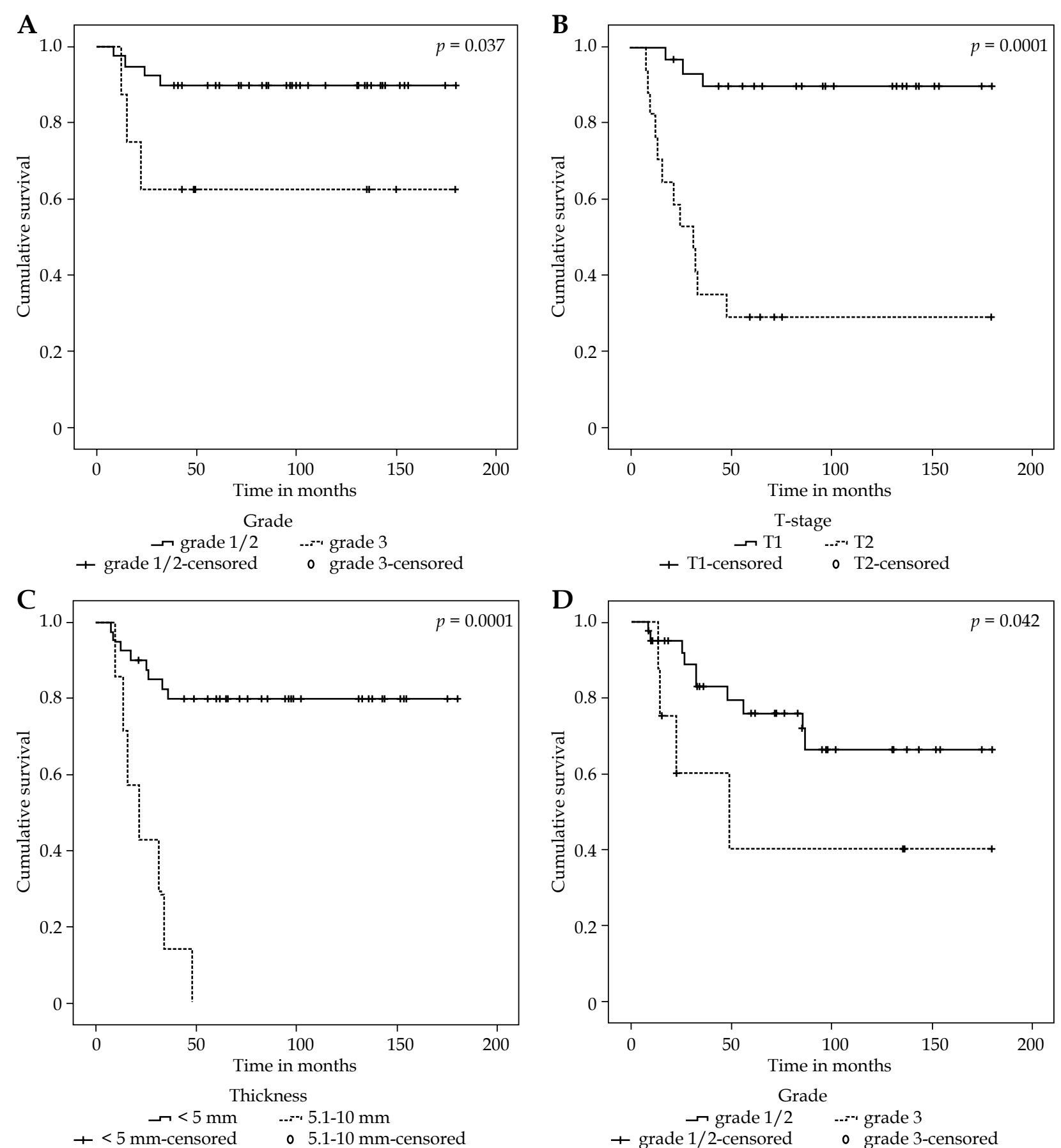

Fig. 2. Brachytherapy only group. A) Local recurrence grade 1 and 2 vs. grade 3. B) Nodal recurrence T1 vs. T2. C) Nodal recurrence $\leq 5 \mathrm{~mm}$ vs. $>5 \mathrm{~mm}$ to $10 \mathrm{~mm}$. D) Disease-free survival grade 1 and 2 vs. grade 3 
tients $(p=0.037$ ) (Figure 2A). The nodal recurrence-free rate at 6 years in T1 patients was $89.8 \%$ vs. $29.4 \%$ in T2 patients $(p=0.0001)$ (Figure 2B). The nodal recurrence-free rate at 6 years in patients with tumor thickness $\leq 5 \mathrm{~mm}$ was $79.7 \%$ vs. $0 \%$ in patients with tumor thickness between $5.1 \mathrm{~mm}$ and $10 \mathrm{~mm}(p=0.0001)$ (Figure 2C). Disease-free survival at 6 years in tumor histologic grade 1 and 2 patients was $76.3 \%$ vs. $40 \%$ in grade 3 patients $(p=0.042$ ) (Figure 2D).

The local recurrence-free rates in HDR-BT and PB at 6 years was $85.1 \%$ and $100 \%$, respectively $(p=0.044)$ (Figure $3 \mathrm{~A})$. Nodal recurrence-free rate at 6 years was $65.5 \%$

A

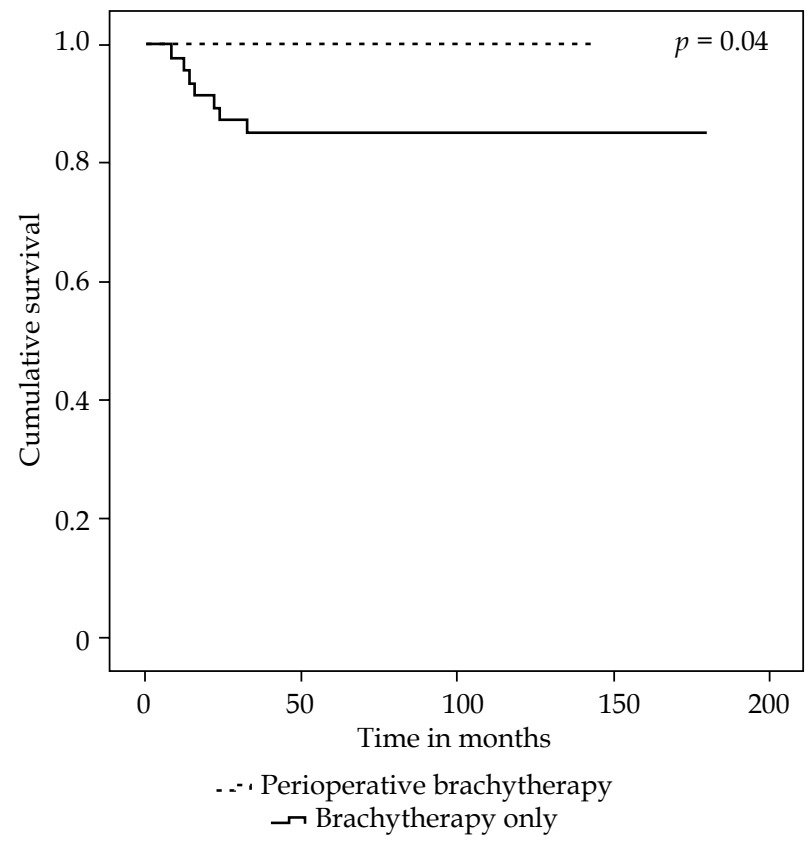

C

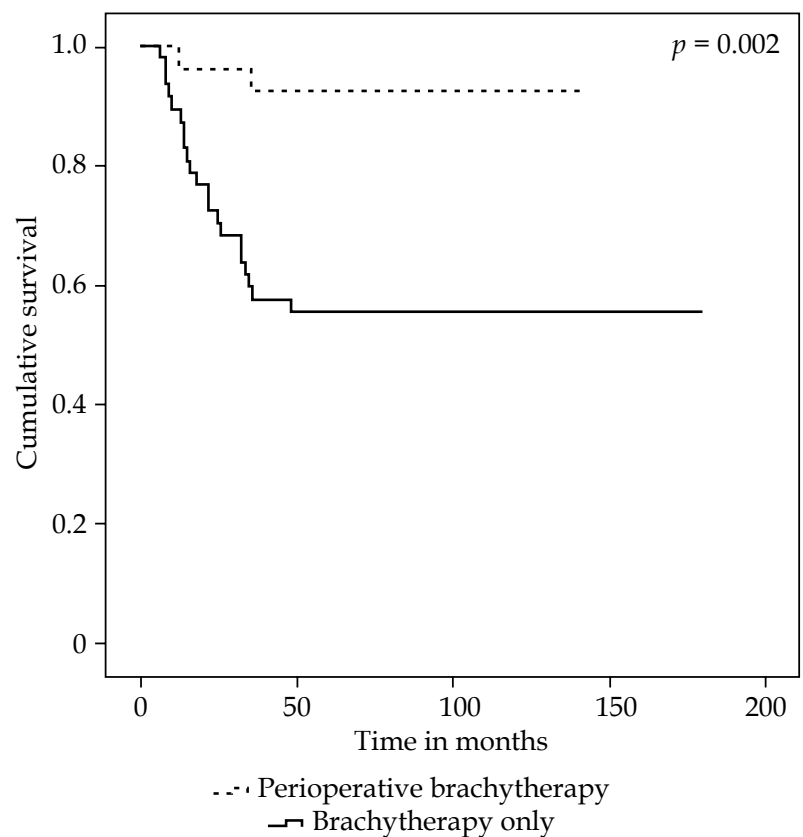

in HDR BT group, and $96.2 \%$ in PB group $(p=0.005)$ (Figure $3 \mathrm{~B})$. Disease-free survival rate at 6 years was $55.3 \%$ and $92.3 \%$, respectively, in HDR-BT and $\mathrm{PB}$ group, respectively $(p=0.001)$ (Figure 3C). The overall survival at 6 years was $74.7 \%$ in HDR-BT group, and $92.3 \%$ in PB group $(p=0.032)$ (Figure 3D). Six patients $(8.2 \%)$ died of disease, while eleven patients $(15 \%)$ died due to other causes. None of the patients in either group developed a local or regional recurrence after 48 months.

The Cox proportional Hazard model was used for multivariate analysis (Table 3 ). Tumor grade was a signif-

B

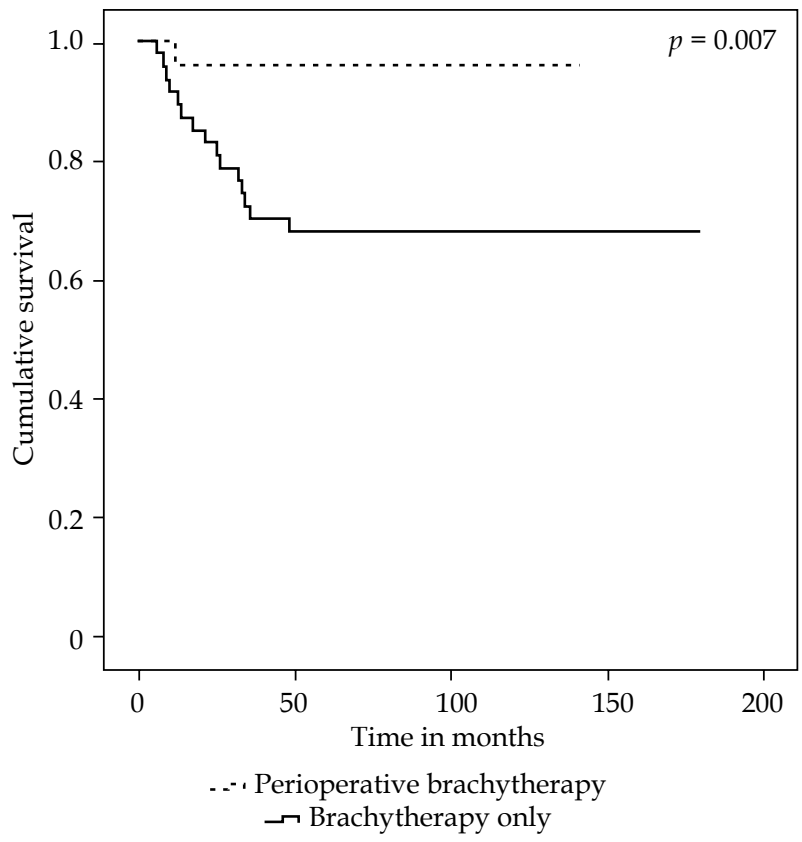

D

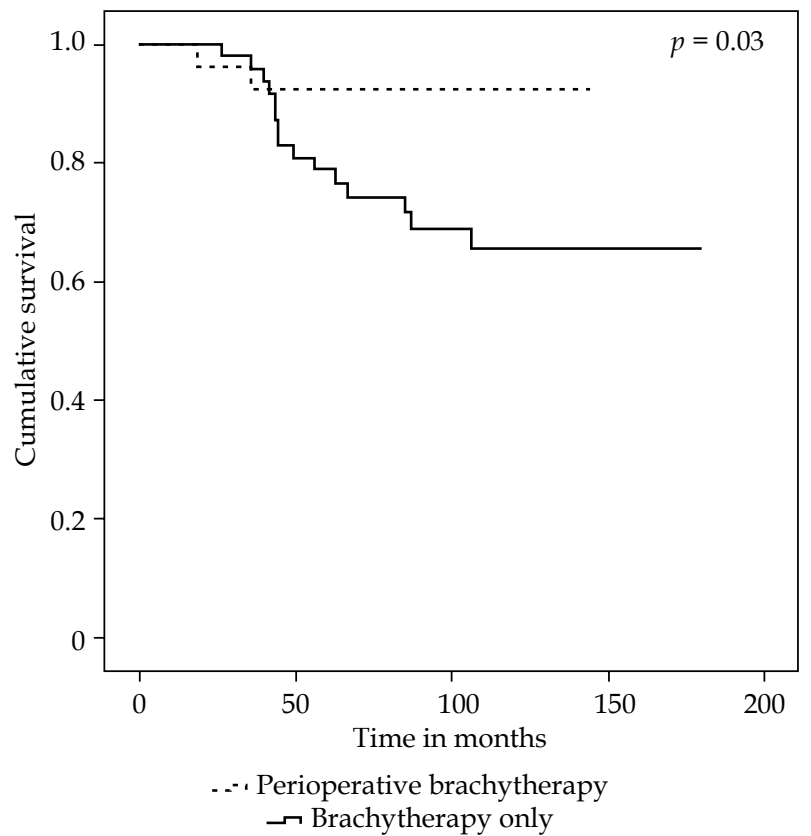

Fig. 3. Perioperative brachytherapy vs. brachytherapy only group. A) Local recurrence. B) Nodal recurrence. C) Disease-free survival. D) Overall survival 
Table 3. Prognostic factors for loco-regional recurrence and disease-free survival in high-dose-rate brachytherapy (HDR-BT) group

\begin{tabular}{|c|c|c|c|c|c|c|c|}
\hline \multirow{2}{*}{$\begin{array}{l}\text { Prognostic } \\
\text { factor }\end{array}$} & \multirow{2}{*}{$\begin{array}{l}\text { Number of } \\
\text { patients in } \\
\text { each category }\end{array}$} & \multicolumn{2}{|c|}{ Local recurrence } & \multicolumn{2}{|c|}{ Nodal recurrence } & \multicolumn{2}{|c|}{ Disease-free survival } \\
\hline & & Univariate & Multivariate & Univariate & Multivariate & Univariate & Multivariate \\
\hline Age & 47 & $p=0.448$ & $p=0.270$ & $p=0.689$ & $p=0.500$ & $p=0.011$ & $p=0.003$ \\
\hline \multicolumn{8}{|l|}{ Gender } \\
\hline Male $=0$ & 31 & $p=0.533$ & $p=0.252$ & $p=0.955$ & $p=0.660$ & $p=0.845$ & $p=0.290$ \\
\hline Female $=1$ & 16 & & & & & & \\
\hline \multicolumn{8}{|l|}{ T-stage } \\
\hline $\mathrm{T} 1=0$ & 30 & $p=0.679$ & $p=0.340$ & $p<0.001$ & $p=0.007$ & $p=0.077$ & $p=0.026$ \\
\hline $\mathrm{T} 2=1$ & 17 & & & & & & \\
\hline \multicolumn{8}{|l|}{ Grade } \\
\hline $\mathrm{G} 1 / 2=0$ & 39 & $p=0.056$ & $p=0.029$ & $p=0.512$ & $p=0.591$ & $p=0.074$ & $p=0.109$ \\
\hline $\mathrm{G} 3=1$ & 8 & & & & & & \\
\hline \multicolumn{8}{|l|}{ Thickness } \\
\hline$\leq 5 \mathrm{~mm}=0$ & 40 & $p=1.000$ & $p=0.480$ & $p<0.001$ & $p=0.154$ & $p=0.067$ & $p=0.914$ \\
\hline $5.1-10 \mathrm{~mm}=1$ & 7 & & & & & & \\
\hline
\end{tabular}

icant prognostic factor in predicting local recurrence $(p=$ $0.029, \mathrm{HR}=6.36, \mathrm{CI}: 1.21-33.44)$ on multivariate analysis. Tumor thickness and T-stage were significant in univariate analysis for nodal recurrence $(p=0.0001)$. However, on multivariate analysis, only T-stage was a significant predictor of nodal recurrence. Age as a continuous variable $(p=0.003, \mathrm{HR}=1.08$; CI: 1.03-1.15) and T-stage $(p=$ $0.026, \mathrm{HR}=0.15$; CI: 0.03-0.80) were the only significant predictors of disease-free survival. In patients for whom CT-based planning was done, the median coverage index, quality index, and uniformity index was 0.92 (range, $0.68-0.92$ ), 1.32 (range, 1.21-1.58), and 1.34 (range, 1.251.67), respectively. Only $72 \%$ patients achieved coverage index greater than $90 \%$. Grade 3 mucositis was the only

A

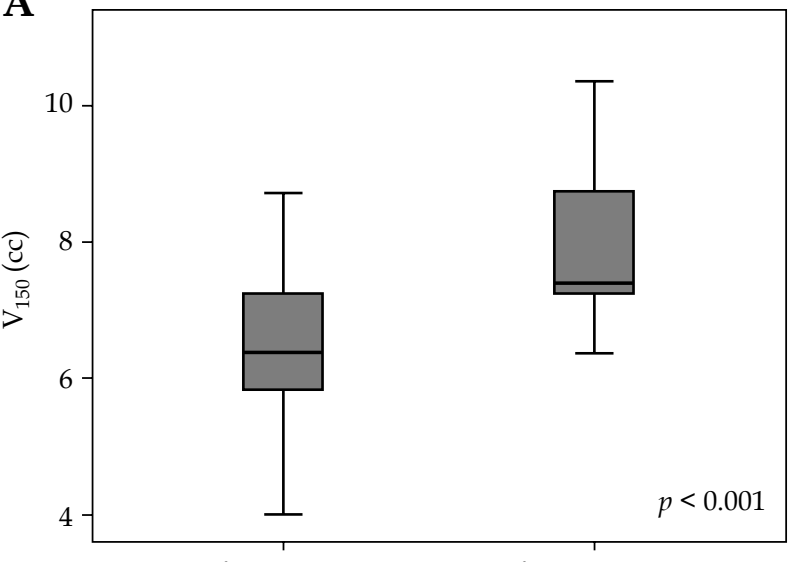

No soft tissue necrosis Soft tissue necrosis early complication seen in patients by the end of second week post-implant. No patient developed grade 4 acute complication. Acute mucositis settled within a median period of 3 months with conservative management.

Twelve patients $(16 \%)$ developed soft tissue necrosis (STN), which settled with conservative management. Five patients $(7 \%)$ developed osteoradionecrosis (ORN), out of which one patient had to undergo surgery. In the other 4 patients, even though the bone remained exposed, a complete healing was observed. In the PB group, no patient developed STN or ORN. An independent samples t-test was run to determine the differences in $V_{150}$ values between patients developing STN and ORN. Data is expressed as mean \pm standard deviation, unless oth-

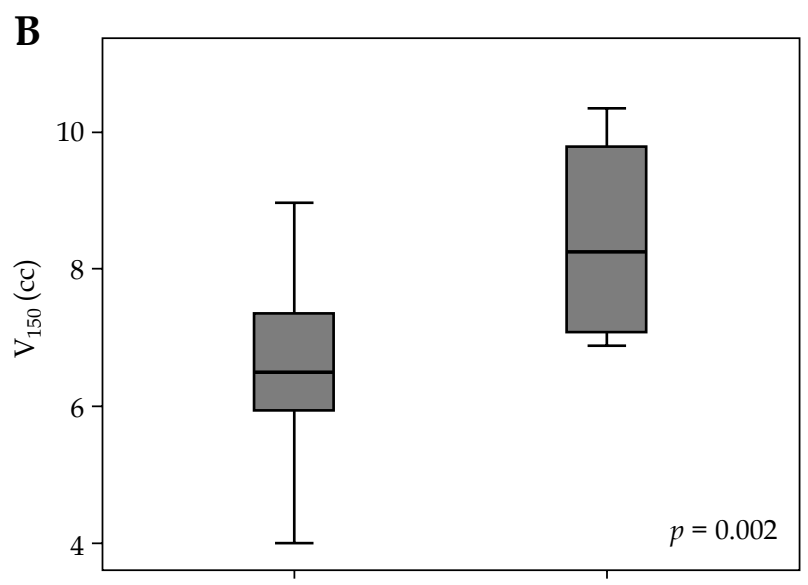

No osteoradionecrosis Osteoradionecrosis

$V_{150}$ - volume of tissue receiving $150 \%$ of the prescribed dose

Fig. 4. $V_{150}$ relation to soft tissue necrosis and osteoradionecrosis 
erwise specified. There were no outliers in the data as assessed by inspection of a box-plot. $\mathrm{V}_{150}$ values in patients developing and not developing STN/ORN were normally distributed as assessed by Shapiro-Wilke's test $(p>0.05)$, and there was homogeneity of variances as assessed by Levene's test for equality of variances $(p=$ 0.268 for STN; $p=0.098$ for ORN). $V_{150}$ values in cc were high in patients developing STN $(7.89 \pm 1.2)$ and ORN $(8.42 \pm 1.64)$. The corresponding values of $\mathrm{V}_{150}$ in patients not developing STN and ORN was $6.52 \pm 0.99$ and 6.64 \pm 1.04 , respectively. There was a statistically significant difference of 1.4 (95\% CI: 0.73-2.02, $p=0.005)$ for STN, and statistically significant difference of 1.8 (95\% CI: 0.67-2.88, $p=0.002$ ) for ORN (Figure 4). Age and number of catheters used did not have any impact on the development of STN and ORN.

\section{Discussion}

The primary goal in treating T1/T2 N0 oral tongue cancer is achieving loco-regional control by surgery or radiotherapy $[12,13]$. Choosing the treatment modality continues to generate heated discussions among surgical and radiation oncologists practicing brachytherapy. Surgery remains the preferred option, as it addresses both local and regional issues. Brachytherapy, despite achieving good local control, has the obvious disadvantage of not addressing the regional nodes. Also, there has been a sharp decline in the number of radiation oncologists skilled in brachytherapy, leading to its diminished use.

The local recurrence rates following surgery alone for tongue primary range from $10-63 \%$ indicating that there is a scope for improvement $[14,15,16]$. Most of the local recurrences are salvageable by re-surgery or radiotherapy. However, in low-resource countries where long-term follow-up is an issue, a local recurrence not detected at the right time can have disastrous consequences. The rationale of adding brachytherapy to tumor bed after surgery was to prevent local recurrences. It is a simple procedure and does not prolong the primary treatment excessively. It may be argued that surgery alone may give equivalent results. However, published literature shows that local recurrences can vary widely from center to center. Parikh et al. [14] reported a local recurrence of $25 \%$ after wide local excision compared to $9 \%$ in hemiglossectomy group in 126 patients who underwent surgery alone for T1/T2 N0 oral tongue cancer. They recommend hemiglossectomy for better survival and decreased local recurrence in early tongue cancer patients. Most T1 tumors may not need adjuvant therapy post-surgery. However, role of PB in allowing smaller resections can be explored in patients who would otherwise require hemiglossectomy. Ganly et al. [15] reported $11 \%$ local recurrences in 216 patients of early stage squamous cell cancer of oral tongue treated at Memorial Sloan-Kettering Cancer Center from 1985 to 2005. A positive surgical margin was the main independent predictor of local recurrence free survival (91\% vs. $66 \%$ for a negative surgical margin; $p=0.0004$ ). Liu et al. [16] reported $63 \%$ local recurrence rate in the first 2 years of follow-up in a group of 26 patients treated with surgery alone in early tongue cancer patients. Shim et al. [6] reported on 86 patients with early tongue cancer managed with surgery alone and surgery with postoperative radiotherapy. There were no local failures in the group that received radiotherapy, however it was not statistically significant. There were 9\% local recurrences. All the recurrences (21\%), loco-regional and distant, occured in higher grade and invasion depth over $0.5 \mathrm{~cm}$ tumors. The rationale of adding brachytherapy to surgery in early tongue cancer was to try to prevent the significant rates of local recurrence after surgery alone, as mentioned in the above data. Recurrences may need further surgery or external beam therapy, which can result in considerable morbidity that can be avoided with perioperative brachytherapy. Majority of T1 patients may not need additional treatment. High-risk patients, like large $\mathrm{T} 1$ ( $>1 \mathrm{~cm}$ thick) and T2 tumors, higher grade tumors, positive margins, and perineural invasion may benefit from brachytherapy in addition to surgery. The problem is information on all these parameters that may not be available at the time of surgery. Wide local excision with modified radical neck dissection, followed by brachytherapy to the tumor bed during perioperative period gives excellent loco-regional control. In our series, none of the 26 patients in $\mathrm{PB}$ group had a local recurrence with a median follow-up of 74 months.

Prospective trials have not given definitive evidence for the role and timing of neck dissection in early T-stage node negative tongue cancers. The importance of treating regional nodes is highlighted by the patterns of relapse when only the tongue primary is addressed. In patients undergoing primary tongue surgery alone without some form of neck dissection, the incidence of nodal metastasis ranges from $18-42 \%[17,18,19]$. In patients undergoing elective neck dissection, the incidence of metastatic cervical lymph nodes is reported between $28-34 \%$ [20,21]. Shibyua et al. [22] reported 32\% nodal relapse in $\mathrm{T} 1$ and $42.9 \%$ failures in T2 tumors with brachytherapy alone when the neck was not treated. However, in our series of 26 patients who underwent elective neck dissection, histopathology did not reveal any metastatic cervical nodes. This maybe because all the patients had imaging in the form of $\mathrm{CT} /$ ultrasound scan of the neck to assess the lymph nodal status. The most compelling evidence for elective neck dissection over therapeutic neck dissection in early oral cancer comes from a large prospective trial by D'Cruz et al. Elective neck dissection resulted in better overall survival ( $80 \%$ vs. $67.5 \%$ ) and disease-free survival rates $(69.5 \%$ vs. $45.9 \%)$ at 3 years in early oral cancers, when compared to patients who underwent therapeutic neck dissection. The overall survival benefit of elective neck dissection persisted across the subgroups analyzed and in early node negative mobile tongue cancer patients. In many centers, upfront elective neck dissection forms an integral part of the treatment in early tongue cancer with negative neck nodes.

In patients undergoing HDR-BT as the initial treatment, 7 patients (14.8\%) developed local recurrence, all of whom were salvaged with surgery. We used single plane implant, even for T2 tumors. Ideally, only small, T1 tumors should be treated with a single plane, and bigger tumors should be treated with a double plane implant to 
cover the tumor adequately. Single plane implantation should not be done for tumors more than $1 \mathrm{~cm}$ in thickness. The most important prognostic factor in predicting risk of nodal metastasis in early tongue cancer appears to be the invasion of the primary tumor to a thickness of $>5 \mathrm{~mm}$. In the perioperative group, 6 out of 26 patients had a depth of invasion $\geq 4 \mathrm{~mm}$. Deepak et al. found a $38.5 \%$ incidence of lymph nodal metastasis in oral tongue cancers in a retrospective analysis of 343 oral cancers patients, when the tumor thickness was between 4.1$6 \mathrm{~mm}$ in contrast to $11.2 \%$ incidence, when thickness was 2.1-4 mm [23]. Our study also reconfirms the importance of tumor thickness in predicting nodal recurrence. $20 \%$ $(8 / 40)$ patients developed nodal recurrence in $\mathrm{BO}$ group when tumor thickness was $\leq 5 \mathrm{~mm}$. $100 \%$ (7/7) patients developed nodal recurrence when tumor thickness was 5.1 to $10 \mathrm{~mm}$. The local recurrence-free rates in patients with grade 3 tumors $(62.5 \%)$ were inferior to grade 1 and 2 tumors $(89.7 \%)$. This may require a higher dose and additional margin in the form of a double plane implant to decrease local recurrence in grade 3 tumors.

HDR brachytherapy for oral tongue cancer can lead to STN and ORN of mandible, because of proximity of the brachytherapy tubes delivering high dose of radiation to mandible. The incidence of ORN and STN varies from $5.3 \%$ to $20 \%$ in various studies, because of wide variation in fractionation schedules practiced at different institutions. Umeda et al. [24] reported a $20 \%$ incidence of mandibular ORN in 25 stage 1 and stage 2 tongue cancer patients treated with HDR brachytherapy. They found higher local control and incidence of ORN in the HDR group compared to LDR group. Some groups have reported a higher incidence of these complications, when external beam radiotherapy was combined with brachytherapy $[25,26,27,28]$. In our study, $16 \%$ and $7 \%$ patients developed STN and ORN, respectively. All patients with STN improved with conservative management. One patients with ORN required surgery. The relative higher incidence of STN and ORN can be related to the high $V_{150}$ values, which could have been improved with better optimization during treatment planning. The use of a spacer between the brachytherapy cannulas and mandible could have also reduced the incidence of these complications. There was no incidence of STN or ORN in the PB group, probably related to the overall lower total dose delivered.

Role of sentinel lymph node biopsy (SNLNB) in this scenario is an exciting topic. Multiple studies have shown excellent sensitivity (90-100\%) and close to $95 \%$ negative predictive value $[29,30,31]$. Despite its promise, it is yet to be accepted as a standard treatment.

\section{Conclusions}

HDR-BT alone gives acceptable loco-regional control in T1 tumors. Neck needs to be addressed by elective neck dissection or irradiation in T2 stage tumors, because of high incidence of nodal recurrences in patients undergoing HDR-BT alone. Perioperative brachytherapy appears to be promising in view of excellent loco-regional control with minimal morbidity. $\mathrm{PB}$ is in- vestigational and can be considered in certain high-risk cases like T2 tumors, positive margins, and perineural invasion. Molecular markers to identify high-risk disease and SNLNB may be the way forward to determine which patients require neck nodes treatment in early oral tongue cancer.

\section{Acknowledgements}

Ms. Anugraha Mathavan for thorough proofreading of the article.

\section{Disclosure}

The authors report no conflict of interest.

\section{References}

1. Jemal A, Bray F, Center MM et al. Global Cancer Statistics. CA Cancer J Clin 2011; 61: 69-90.

2. Warnakulasuriya S. Global epidemiology of oral and oropharyngeal cancer. Oral Oncol 2009; 45: 309-316.

3. Fein DA, Mendenhall WM, Parsons JT et al. Carcinoma of the oral tongue: a comparison of results and complications of treatment with radiotherapy and/or surgery. Head Neck 1994; 16: 358-365.

4. Pernot M, Malissard L, Hoffstetter S et al. The study of tumoral, radiobiological, and general health factors that influence results and complications in a series of 448 oral tongue carcinomas treated exclusively by irradiation. Int J Radiat Oncol Biol Phys 1994; 29: 673-679.

5. D'Cruz AK, Vaish R, Kapre $\mathrm{N}$ et al. Elective versus therapeutic neck dissection in node negative oral cancer. $N$ Engl J Med 2015; 373: 521-529.

6. Shim SJ, Cha J, Koom WS et al. Clinical outcomes for T12N0-1 oral tongue cancer patients underwent surgery with and without postoperative radiotherapy [serial online]. $R a$ diat Oncol 2010; 5: 43.

7. Yuen AP, Ho CM, Chow TL et al. Prospective randomized study of selective neck dissection versus observation for N0 neck of early tongue carcinoma. Head Neck 2009; 31: 765-772.

8. Al-Rajhi N, Khafaga Y, El-Husseiny J et al. Early stage carcinoma of oral tongue: prognostic factors for local control and survival. Oral Oncol 2000; 36: 508-514.

9. Kligerman J, Lima RA, Soares JR et al. Supraomohyoid neck dissection in the treatment of T1/T2 squamous cell carcinoma of the oral cavity. Am J Surg 1994; 168: 391-394.

10. Hicks WL Jr, North JH Jr, Loree TR et al. Surgery as a single modality therapy for squamous cell carcinoma of the oral tongue. Am J Otolaryngol 1998; 19: 24-28.

11. Sobin LH, Gospodarowicz MK, Wittekind C: UICC: TNM classification of malignant tumours. $7^{\text {th }} \mathrm{ed}$. Wiley-Blackwell, Oxford 2009.

12. Spiro RH, Strong EW. Epidermoid carcinoma of the mobile tongue-treatment by partial glossectomy alone. Am J Surg 1971; 122: 707-710

13. Decroix Y, Ghossein NA. Experience of the Curie Institute in treatment of cancer of the mobile tongue. Cancer 1981; 47: 496-502.

14. Parikh HK, Rao RS, Sukhthankar P et al. Surgery in early cancer of the oral tongue (T1-2). Wide excision versus hemiglossectomy. Indian J Otolaryngol Head Neck Surg 1998; 50: 349-353.

15. Ganly I, Patel S, Shah J. Early stage squamous cell cancer of the oral tongue - clinicopathologic features affecting outcome. Cancer 2012; 118: 101-111. 
16. Liu JC, Sopka DS, Mehra R et al. Early oral tongue cancer initially managed with surgery alone: Treatment of recurrence. World J Othorhinolaryngol Head Neck Surg 2016; 2: 193-197.

17. Urashima Y, Nakamura K, Shioyama Y et al. Treatment of early tongue carcinoma with brachytherapy: results over a 25-year period. Anticancer Res 2007; 27: 3519-3523.

18. Vermund $\mathrm{H}$, Brennhovd I, Kaalhus $\mathrm{O}$ et al. Incidence and control of occult neck node metastases from squamous cell carcinoma of the anterior two-thirds of the tongue. Int J Radiat Oncol Biol Phys 1984; 10: 2025-2036.

19. Cunningham MJ, Johnson JT, Myers EN et al. Cervical lymph node metastasis after local excision of early squamous cell carcinoma of the oral cavity. Am J Surg 1986; 152: 361-366.

20. Keski-Säntti H, Atula T, Törnwall J et al. Elective neck treatment versus observation in patients with $\mathrm{T} 1 / \mathrm{T} 2 \mathrm{~N} 0$ squamous cell carcinoma of oral tongue. Oral Oncol 2006; 42: 96-101.

21. Lim YC, Lee JS, Koo BS et al. Treatment of contralateral N0 neck in early squamous cell carcinoma of the oral tongue: elective neck dissection versus observation. Laryngoscope 2006; 116: 461-465.

22. Shibuya H, Hoshina M, Takeda M et al. Brachytherapy for stage I \& II oral tongue cancer: an analysis of past cases focusing on control and complications. Int J Radiat Oncol Biol Phys 1993; 26: 51-58.

23. Balasubramanian D, Ebrahimi A, Gupta R et al. Tumor thickness as a predictor of nodal metastasis in oral cancer: Comparison between tongue and floor of mouth subsites. Oral Oncol 2014; 50: 1165-1168.

24. Umeda M, Komatsubara H, Nishimatsu $\mathrm{N}$ et al. High-dose rate interstitial brachytherapy for stage I-II tongue cancer. Oral Surg Oral Med Oral Pathol Oral Radiol Endod 2000; 90: 667-670.

25. Tsai CJ, Hofstede TM, Sturgis EM et al. Osteoradionecrosis and radiation dose to the mandible in patients with oropharyngeal cancer. Int J Radiat Oncol Biol Phys 2013; 85: 415-420.

26. Fujita M, Hirokawa $Y$, Kashiwado K et al. An analysis of mandibular bone complications in radiotherapy for $\mathrm{T} 1$ and T2 carcinoma of the oral tongue. Int J Radiat Oncol Biol Phys 1996; 34: 333-339.

27. Wendt CD, Peters LJ, Delclos L et al. Primary radiotherapy in the treatment of stage I and II oral tongue cancers: Importance of the proportion of therapy delivered with interstitial therapy. Int J Radiat Oncol Biol Phys 1990; 18: 1287-1292.

28. Reuther T, Schuster T, Mende U et al. Osteonecrosis of the jaws as a side effect of radiotherapy of the head and neck tumor patients - a report of a thirty-year retrospective review. Int J Oral Maxillofac Surg 2003; 32: 289-295.

29. Civantos FJ, Zitsch RP, Schuller DE et al. Sentinel lymph node biopsy accurately stages the regional lymph nodes for T1-T2 oral squamous cell carcinomas. Results of a prospective multi-institutional trial. J Clin Oncol 2010; 28: 1395-1400.

30. Ross GL, Soutar DS, Gordon MacDonald D et al. Sentinel lymph node biopsy in head and neck cancer: preliminary results of a multicenter trial. Ann Surg Oncol 2004; 11: 690-696.

31. Schilling C, Stoeckli SJ, Haerle SK et al. Sentinel European Node Trial (SENT): 3-year results of sentinel node biopsy in oral cancer. Eur J Cancer 2015; 51: 2777-2784. 